\title{
Laparoscopic findings in chronic pelvic pain
}

Bajracharya J', Shrestha NS², Karki C³, Saha R ${ }^{4}$

${ }^{1}$ Lecturer, ${ }^{2}$ Associate Professor, ${ }^{3}$ Professor and Head of department, ${ }^{4}$ Associate Professor, Department of Obstetrics and Gynaecology, Kathmandu Medical College Teaching Hospital, Kathmandu, Nepal

\section{Abstract}

Background: Chronic pelvic pain is a common problem in reproductive age group women. Diagnosis of chronic pelvic pain needs multidisciplinary approach. Diagnostic laparoscopy is one of the investigations which can help in reaching the diagnosis.

Objective: To know the etiology in chronic pelvic pain.

Methods: This was a descriptive study done in the Department of Obstetrics and Gynaecology of Kathmandu medical college teaching hospital from January 2010 to June 2012 (30 months). All the cases of laparoscopic surgery done for chronic pelvic pain were noted and details of these cases were analyzed regarding age, parity and laparoscopic findings. Results: Total 48 cases of Chronic Pelvic Pain underwent diagnostic laparoscopy during the study period. Mean age of cases were 33 years, ranging from $20-46 y$ rs. Almost half of the cases $43.75 \%$ were of parity two. Laparoscopic finding was negative in $29.17 \%$ and pelvic pathology was present in $70.83 \%$ of the cases. Out of the pelvic pathology endometriosis was present in $55.88 \%$ followed by pelvic adhesions, pelvic congestion and pelvic inflammatory disease, chronic ectopic in $20.58 \%, 14.70 \%, 5.88 \%, 2.94 \%$ respectively.

Conclusion: Diagnostic laparoscopy is a useful modality in the diagnosis of etiology and management of Chronic pelvic pain. In our study, Pelvic endometriosis was the most common pelvic pathology in cases of Chronic pelvic pain.

Key words: Chronic pelvic pain, Diagnostic laparoscopy, Endometriosis

\section{INTRODUCTION}

$\mathbf{P}$ ain is an unpleasant sensory or emotional experience associated with actual or potential damage ${ }^{1}$ whereas Chronic pelvic pain (CPP) is an intermittent or constant pain in the lower abdomen or pelvis of at least six months duration not occurring exclusively with menstruation or intercourse and not associated with pregnancy ${ }^{2}$.

CPP is a symptom, not a disease. It is one of the most common symptoms in patients attending Gynaecology outpatient department. CPP afflicts 5-10\% of women ${ }^{3}$. Prevalence of CPP has been reported as $3.8 \%$ in women aged 15-73 which is higher than the prevalence of migraine $(2.1 \%)^{4}$. CPP is common problem and presents a major challenge to the gynaecologist and other health care providers because of its unclear etiology, complex natural history and poor response to therapy.

\footnotetext{
Address for correspondence

Junu Bajracharya

Lecturer, Department of Obstetrics and Gynaecology,

Kathmandu Medical College Teaching Hospital,

Kathmandu, Nepal

E-mail:dr_junu@hotmail.com
}

Diagnosis of the cause of CPP requires multidisciplinary approach as it may arise from any structure related to pelvis including abdominal and pelvic wall.

Various causes of CPP have been identified which are dysmotility disorders including irritable bowel syndrome (50-80\%), musculoskeletal disorder (30-70\%), urological cause (5-10\%), endometriosis and dense adhesion $<5 \%$, multiple medical diagnosis $<5 \%$ and $40-50 \%$ of woman with CPP had history of physical and sexual abuse ${ }^{5}$. For the diagnosis of CPP initially proper detailed history of pain, its quality, location, radiation and duration, aggravating and relieving factor, bowel and bladder habits and its impact on the activities of daily living should be considered. Beside this, history of physical and sexual abuse should be taken. Thorough examination and investigation should be done for diagnosis of chronic pelvic pain.

Laparoscopy is one of the investigations which help us in reaching the diagnosis of chronic pelvic pain. It does not only establish the diagnosis but also can modify the treatment. 
This study is an attempt to understand the etiology of this complex problem in Nepalese women.

\section{METHODS}

This is a descriptive study done in the Department of Obstetrics and Gynaecology of Kathmandu medical college teaching hospital from January 2010 to June 2012. All the cases of CPP admitted for diagnostic laparoscopy were noted from admission records. Thorough history including demographic details were noted. They were followed up after diagnostic laparoscopy and their laparoscopic finding were also noted. Diagnostic laparoscopy was performed under General anaesthesia. Ten millimeter supraumbilical port was made by Hasson's technique and carbon dioxide pneumoperitonem was created. Second port of five $\mathrm{mm}$ was created in left side lateral to left rectus muscle under direct vision avoiding the injury to the inferior epigastric vessel .Diagnostic laparoscopy was performed by standard technique and laparoscopic findings were noted. A five $\mathrm{mm}$ port was made approximately five centimeters above the second port in cases where intervention was required.

\section{RESULTS}

Total 247 cases were posted for laparoscopic procedure. Among these 48 cases were done for CPP during the study period. Ages ranged from 20 to 46 years with the mean age of 33 years.

Table 1: Parity of woman who underwent laparoscopy for chronic pelvic pain

\begin{tabular}{|cc|}
\hline Parity & Numbers (percentage) \\
\hline Para1 & $12(25 \%)$ \\
\hline Para2 & $21(43.75 \%)$ \\
\hline Para3 & $12(25 \%)$ \\
\hline Para $>3$ & $3(6.25 \%)$ \\
\hline
\end{tabular}

Table 2: Laparoscopic findings in chronic pelvic pain

\begin{tabular}{|cc|}
\hline Pelvic pathology & $\begin{array}{c}\text { Number of Cases } \\
\text { (Percentage) }\end{array}$ \\
\hline Endometriosis & $19(55.88 \%)$ \\
\hline Pelvic adhesions & $7(20.58 \%)$ \\
\hline Pelvic congestion & $5(14.70 \%)$ \\
\hline PID & $2(5.88 \%)$ \\
\hline Chronic ectopic & $1(2.94 \%)$ \\
& Total 34 \\
\hline
\end{tabular}

Table 1 shows that 21 out of 48 i.e. almost half of the cases (43.75\%) were of parity two. As shown in Table 2, out of 48 cases, 34 (70.83\%) cases had pelvic pathology in which endometriosis was the commonest followed by adhesion, pelvic congestion and pelvic inflammatory disease.

\section{DISCUSSION}

CPP, which is one of the major challenges for health professional, needs multidisciplinary approach for care and management of the cases. Proper history, examination should be considered in case of CPP6. Diagnostic laparoscopy is one of the gold standards in the diagnosis of CPP ${ }^{16}$. By laparoscopic procedure, not only diagnosis but also therapeutic procedure can be done in the same setting. In this study, the cause for CPP was identified in $70.83 \%$ which made the management of cases specific and targeted. Chhetri $S$ et al in Diagnostic laparoscopy was able to detect pathology in $45(81.8 \%)$ patients ${ }^{6}$. Similarly study done by Swanton et al shows positive finding in $90 \%^{7}$. Mara et al shows positive diagnostic laparoscopy in $82.3 \%{ }^{8}$, Cunnanan et al detected pathology in $82.5 \%{ }^{9}$, Marana et al in $80 \%{ }^{10}$, Kantoravdis et al in $76 \%{ }^{11}$ and Shripad Hebbar et al shows pelvic pathology in $58 \%{ }^{12}$. Thus laparoscopy helps us to find out the cause of chronic pelvic pain which would be missed if we did not do diagnostic laparoscopy.

Various cause of CPP had been identified in different study done at different places. In our study endometriosis was the commonest cause of chronic pelvic pain which accounted for $52.63 \%$ followed by adhesion, congestion, pelvic inflammatory disease and chronic ectopic $21.06 \%, 15.79 \%, 5.26 \%$, and $5.26 \%$ respectively. In a study by Howard FM et al endometriosis was present in 40 percent of the cases followed by pelvic adhesions, chronic pelvic inflammatory disease, and ovarian cysts ${ }^{4}$. Similarly Razia Iftikar showed $56.6 \%$ endometriosis followed by pelvic adhesion in $16.6 \%, 6.6 \%$ benign ovarian cyst and PCOD ${ }^{14}$. Sharma D et al showed that commonest finding on laparoscopy was adhesions in $40 \%$, endometriosis in $18 \%$, and pelvic congestion syndrome in $20 \%{ }^{15}$. Similarly in a study done by Chhetri $\mathrm{S}$, diagnostic laparoscopy was able to detect pathology in $45(81.8 \%)$ patients. Pelvic adhesions was the most common cause of chronic pelvic pain which was present in $16(29 \%)$ of the women, followed by PID in (12.7\%), endometriosis in $(9.1 \%)$, pelvic congestion $(7.2 \%)$, pelvic tuberculosis (7.2\%), fibroid uterus (7.2\%), ovarian cysts in (7.2\%) and parafimbrial cyst in $3.4 \%{ }^{7}$.

Shripad Hebbar et al revealed that the most common pelvic pathology by laparoscopy was pelvic adhesions (20.9\%), followed by pelvic congestion $18.6 \%{ }^{13}$. Newham AP et al, shows that $16 \%$ of women had endometriosis and pelvic adhesions in $40 \%{ }^{16}$. Previously it was said that 
endometriosis is a disease of developed countries rather than the developing countries. In developing countries, in past there used to be trend of early child bearing, multiple child bearing, prolonged breast feeding but the trend is changing nowadays. Probably this is the reason why high prevalence of endometriosis is seen in this study.

\section{CONCLUSION}

Endometriosis was found to be one of the most common causes for chronic pelvic pain in this study. Diagnostic laparoscopy should be considered in all the cases of chronic pelvic pain as it is a useful modality in the diagnosis and treatment of chronic pelvic pain.

9. Mara M, Fucikova Z, Kuzel D, Dohnalova A, Haakova L, Zivny J. Laparoscopy in chronic pelvic pain-a retrospective clinical study. Ceska Gynekol. 2002;67(1):38-46.

10. Cunanan RG, Norman MD, Courey NG, Lippes J. Laparoscopic findings in patients with pelvic pain. Am J Obstet Gynecol. 1983;146:589-591.

11. Marana R, Paielle FV, Muzii L, Dell'Acqua S, Mancuso $\mathrm{S}$. The role of laparoscopy in the evaluation of Chronic Pelvic Pain. Minerva Ginecol. Jun 1993;45(6):281-86.

12. Kontoravdis A, Hassan E, Hassiakos D, Botsis D, KontoravdisN, Creatsas G. Laparoscopic evaluation and management of chronic pelvic pain during adolescence. Clin Exp Obstet Gynecol. 1999;26(2):7677.

13. Hebbar S, Chawla C. Role of laparoscopy in evaluation of chronic pelvic pain. J Minim Access Surg. September 2005;1(3):116-120.

14. Razia iftikhar. Outcome of laparoscopy in chronic pelvic pain. JSP (International). 2008;13(4):155-158.

15. Sharma D, Dahiya K, Duhan N, Bansal R. Diagnostic laparoscopy in chronic pelvic pain. Arch Gynecol Obstet. Feb 2011;283(2):295-7.

16. Newham AP, van der Spuy ZM, Nugent F. Laparoscopic findings in women with chronic pelvic pain. S Afr Med J. 1996 Sep;86(9 Suppl):1200-3. 